\title{
Research on Combined Rehabilitation Method and Its Mechanism of Traditional Chinese Medicine Daoyin Technique with Biofeedback Technique
}

\author{
Wenru Zhao ${ }^{1,2}$ (D), Pengchuan Zhang1, Ming Qi1 ${ }^{1}$, Yuru Song ${ }^{1}$ \\ ${ }^{1}$ Neurological Training and Rehabilitation Research Room, Hospital of Traditional Chinese and \\ Western Medicine, Beijing, China; ${ }^{2}$ Branch of Neurological Training Rehabilitation Technique, \\ China Association of Information of Traditional Chinese Medicine, Beijing, China
}

Correspondence to: Wenru Zhao, zhaowenru7006@sina.com, zhaowenru@hotmail.com Keywords: Rehabilitation, Daoyin Technique of Traditional Chinese Medicine, Biofeedback, Hemiplegia, Elbow Extension Function

Received: November 7, $2019 \quad$ Accepted: December 13, $2019 \quad$ Published: December 16, 2019

Copyright $\odot 2019$ by author(s) and Scientific Research Publishing Inc.

This work is licensed under the Creative Commons Attribution-NonCommercial International License (CC BY-NC 4.0).

http://creativecommons.org/licenses/by-nc/4.0/

\section{Open Access}

\section{ABSTRACT}

Objective: To study an anti resistance active exercise training method which is consistent with the principle of "transcendental goal power" to promote the plasticity change of central nervous system (CNS) in order to recover the motor function after CNS injury, observe its clinical effect and explore its mechanism. Methods: The potential of CNS was induced by six-step Daoyin technique of Chinese medicine, and the potential signal was received in real time by bio-electrical signal receiving equipment and displayed in curve form. Combined with the Operant conditioning biofeedback technique, targeted guidance was given to patients to continuously enhance the action muscle signal intensity and weaken the antagonistic muscle signal intensity, so that the motor program was gradually re-established. Sixty-four patients with hemiplegic elbow dysfunction of old cerebral infarction who had been trained but could not be improved further and had a history of more than 1 year were treated for one course, 90 times and 45 minutes each time. Lovett, Elbow Rom, Fugl-Meyer, program signal intensity and proportion were evaluated before and after treatment. The data were analyzed statistically. Before and after treatment, Lovett MMT, ROM of elbow joint, Fugl Meyer, motor program signal intensity and proportion were assessed, and the data were statistically analyzed. Results: After treatment and before treatment, there was a significant difference between the functional evaluation data $(P<0.01)$. The function of elbow joint was improved; this is the result of reconstructing the motor program on the basis of developing the potential of CNS by means of the "transcendental goal power" mechanism of the method. There was no recurrence after 6 months of follow-up. Conclusion: The Chi- 


\section{nese Medicine Daoyin feedback rehabilitation technique is an effective method to improve elbow joint function of hemiplegia patients with cerebral infarction.}

\section{INTRODUCTION}

The incidence of stroke is on the rise, with 270 strokes per 100,000 men and 161 strokes per 100,000 women [1]. Of these, the elderly constitute the majority, accounting for more than 40 per cent of the total elderly population [2]. The central nerve cells lack the ability of regeneration, and the function lost after the injury depends on the normal cells and conduction channels around the training injury area to play a functional replacement, that is, the development and utilization of the potential of the central nerve. "Six step Daoyin technique" [3] has a better role in developing the potential of central nervous system. After that, it is necessary to establish a connection between the newly activated brain cells and the remaining brain cells in the injured area [4], so that they can work in a consistent and orderly manner, that is, the process of reconstructing normal exercise program. We use bioelectric signal receiving equipment to receive the intensity and proportion of the motor program signal from the cortex motor center to the triceps and biceps brachii when guiding the patients to extend their elbows in real time on the skin surface of muscle parts, and display it on the screen in the form of digital curve in real time. Then according to the mechanism of operant conditioning biofeedback, guidance of the patient to regulate the intensity and proportion of the motor program signal, make it gradually tend to the normal level, and finally improve the motor function.

\section{MATERIALS AND METHODS}

\subsection{General Information}

Subjects: From June 2006 to June 2008, 64 stroke patients with Hemiplegia and limitation of elbow extension were randomly selected according to their hospitalization sequence, including 36 males and 28 females. There were 34 cases of right Hemiplegia and 30 cases of left Hemiplegia. There were 36 males and 28 females. There were 34 hemiplegia on the right side and 30 hemiplegia on the left side. The age range is 30 - 70 years old, with an average of 52.6 years old. In 64 cases, there were 24 patients with cerebral hemorrhage and 40 patients with cerebral infarction. The course of disease was $1-5$ years, with an average of 3.62 years. Inclusion criteria: 1) patients who met the diagnostic criteria of the National Conference on cerebrovascular diseases in 1995, were definitely diagnosed as cerebral infarction or cerebral hemorrhage by CT or MRI, and were accompanied with hemiplegic upper extremity elbow extension activity limitation; 2) no obvious mental retardation (MMSE score $\geq 20$ points); 3) no serious contracture of elbow joint, and the passive range of activity is basically normal; 4) no significant functional improvement after present use rehabilitation treatment in a regular hospital.

\subsection{Exclusion Criteria}

1) Patients with severe heart, lung and other organ dysfunction, serious infectious diseases, fractures, and other diseases that can not be rehabilitated; 2) Serious cognitive impairment or long-term use of drugs such as sedatives, antidepressants and diazepam, which can not cooperate with the treatment and affect the EMG signal examination; 3) There are frequent epileptics; 4) There are other diseases that affect the movement, such as various myopathy, bone joint and peripheral nerve injury; 5) The treatment is interrupted due to other reasons, and the treatment can not be insisted on for 90 times.

\subsection{Method}

In a warm, quiet room, the patient sits in front of the monitor. The Neuroeducator III biofeedback training instrument and $3 \mathrm{M} \mathrm{Ag} \mathrm{AgCl}$ surface electrode produced by American therapeutic Alliance company were used. Electrode placement of triceps brachii: the first surface electrode was placed on the skin 
surface of the corresponding part of triceps brachii muscle, $6 \mathrm{~cm}$ above the elbow joint plane; vertically upwards $12 \mathrm{~cm}$ Put the second electrode and place the ground electrode between the two electrodes. The surface electrode is connected to the first lead of the biofeedback instrument through a wire. Electrode placement of biceps brachii: The first surface electrode is placed on the skin surface of the corresponding part of the biceps brachii muscle on the $4 \mathrm{~cm}$ above the cubital fossa, the second electrode is placed vertically upward $6 \mathrm{~cm}$, and the grounding electrode is placed between the two electrodes. The surface electrode is connected to the third lead of the biofeedback instrument through a wire. The therapist uses six steps of traditional Chinese medicine guidance to guide the patients to adjust their mind and breath, and then carry out active joint activities of elbow extension and elbow flexion. At the highest point of joint activities, the therapist appropriately increases resistance and tries to encourage the patients to persist for six seconds. The highest signals from the collected motor center to triceps and biceps are taken as the initial data, and two lines are made based on this data. The patients were guided to try to make the signal intensity of triceps brachii exceed the baseline level and biceps brachii lower than the baseline level. In the course of treatment, when the signal intensity of triceps brachii is about to reach the highest point, the therapist creates an emergency state for the patient with words such as "insist, otherwise fall, and then high point", so as to make the signal intensity of triceps brachii exceed the baseline height as much as possible, and pay attention to reduce or not increase the signal intensity of biceps brachii. Then, with the new highest point as the baseline, the patients were guided by the same method to try to make the new triceps signal exceed the new baseline level, the biceps signal further lower than the new baseline level, and so on.

\subsection{Evaluation Method}

The international standard of Lovett MMT, ROM, Fugl Meyer's upper limb motor function, triceps brachii and biceps brachii signal (EMG) intensity and proportion were evaluated at the beginning and the end of treatment, collect data and enter it into a database for analysis.

\section{Statistical Analysis}

The data results were expressed as mean \pm standard deviation $(\bar{X} \pm S$ ), and analyzed by SPSS12.0 statistical software, and $t$-test self-control analysis before and after treatment.

\section{RESULTS}

See Table 1 for the analysis results of muscle strength, ROM (extension angle), Fugl Meyer upper extremity motor function score, motor program signal and other function evaluation data of 64 patients before and after treatment. Before and after treatment, there were significant differences in the intensity of motor program signal, muscle strength, Fugl Meyer and the degree of motion of the joint $(\mathrm{P}<0.01)$. There was no significant difference between the two groups $(\mathrm{P}>0.05)$. Before treatment, the signal intensity of

Table 1. Statistical analysis of functional assessment data before and after treatment $\bar{X} \pm S$.

\begin{tabular}{ccccc}
\hline Assessment & & Before Treatment & After Treatment & P Value \\
\hline \multirow{2}{*}{ MMT } & Triceps & $2.56 \pm 1.42$ & $3.90 \pm 0.42$ & $<0.01$ \\
& Biceps & $2.1 \pm 1.07$ & $3.74 \pm 6.76$ & $>0.05$ \\
Active ROM & Ext. angle & $5.63^{0} \pm 2.15^{0}$ & $35.60^{0} \pm 7.68^{0}$ & $<0.01$ \\
Fugl-Meyer & & $18.21 \pm 6.38$ & $34.75 \pm 5.30$ & $<0.01$ \\
& Triceps & $51.13 \pm 23.14$ & $158.61 \pm 50.63$ & $<0.01$ \\
Signal $(\mathrm{uV})$ & Biceps & $42.67 \pm 31.17$ & $52.23 \pm 26.24$ & $>0.05$ \\
& Differ. & $8.64 \pm 8.03$ & $106.38 \pm 16.76$ & $<0.01$ \\
\hline
\end{tabular}


biceps brachii was higher than triceps brachii. The ratio between the two was 1.4:1, which was the root cause of the difficulty of elbow extension of hemiplegic side upper limb. After treatment, the signal intensity to triceps brachii was increased significantly $(\mathrm{P}<0.01)$. Although the signal intensity to biceps brachii was also increased, there was no significant difference between before and after treatment $(\mathrm{P}>0.05)$. Although the signal intensity to biceps brachii was also increased, there was no significant difference between before and after treatment $(\mathrm{P}>0.05)$. After treatment, there was a significant difference in the motor program signal between biceps and triceps $(\mathrm{P}<0.01)$. The main reason for the improvement of elbow joint's autonomic activity was that the ratio between the two improved from 1.4:1 before treatment to 1:4.1. All patients were followed up for 6 months without recurrence.

\section{DISCUSSION}

\subsection{The Coordinated and Orderly Work of Central Nerve Cells Is the Basis of Motor Function Recovery}

The completion of any joint autonomous movement is the result of the joint work of many central nerve cells. Research shows that the number of central nerve cells needed to control fine movements is far greater than that needed to control gross movements [5]. For example, the amount of motor center cells needed to control a thumb is equivalent to that needed to control a lower limb [6]. The premise for so many cells to dominate a joint activity is their close cooperation and coordinated work.

Although the central nervous cells lack the ability of regeneration, the CNS has the same ability of compensation as other tissues, which is achieved through the plasticity [7] of the CNS. After the CNS injury, the lost cells need to be supplemented from three aspects to restore the original motor control function: 1) the cells in the non dead and semi dark areas of the injured area recovered with the change of the internal environment; 2) normal cells around the injury area, but the function can only be obtained through training; 3 ) regenerated central nerve cells. Due to the lack of regeneration ability of central nerve cells, the number of regeneration is very small, which plays a little role in the recovery of motor function. However, no matter which kind of cells, because the original relationship between cells has changed, in order to achieve their coordinated and orderly working ability, it is necessary to establish new connections to restore the possibility of motor function. Therefore, the recovery of the coordinated and orderly work between cells is the basis of the recovery of motor function, and the training of the recovery of the coordinated and orderly work between cells is the process of reconstructing the motor program in the motor center.

\subsection{The Autonomous Movement of Human Body Is the Result of the Control and Control of the Motor Program [8] in the Motor Center of Cortex}

The formation of the motor program in the human motor center comes from two aspects: 1) heredity: a small part; 2) acquirement: the vast majority. It's a motor program learned after birth. The motor program is the electrical signal from the motor center of cortex, which controls the single joint activity or the various movements completed by multiple joints. Every autonomous movement of human body can only be completed under the control of the motor program in the cortex motor center, and the perfection of the motor program determines the normal degree of the autonomous movement mode. Athletes can improve their competitive level through training, which may be related to improving the precision of motor program and muscle strength.

\subsection{Digital and Objective Display of Motor Program Signal Is an External Condition Necessary for Correcting Disorder Motor Program and Reconstructing Normal Motor Program}

After the injury of central nerve cells, the exercise program will be destroyed in different degrees. The complete destruction of exercise program will lead to the complete loss of exercise, and the lack of coordination between partially destroyed or compensated cells will lead to different degrees of abnormal exercise 
patterns. Only by re-establishing normal exercise program can the original exercise function be restored.

In this study, the activity of elbow extension is normally that the brain sends strong signals to triceps brachii, and the result of its contraction is elbow extension. At the same time, it also sends weak signals to biceps brachii to maintain a certain tension and increase the stability of elbow in elbow extension. In addition to the decrease of signal intensity, the abnormal movement process after brain injury is often due to the imbalance of signal proportion between triceps and biceps. As a result, it is difficult to produce elbow extension. However, in order to reconstruct the normal motor program on the basis of correcting the disorder motor program, we must see the actual operation of the elbow extension program.

The Daoyin feedback technology of traditional Chinese medicine is to use bioelectrical signal detection equipment to receive and display the dynamic continuous motor program feedback signal on the fluorescent screen in the form of curve when Daoyin patients carry out active elbow extension activities. The dynamic continuous motor program feedback signal plays a role equivalent to, but obviously superior to, the target, making the training targeted. After repeated application of guidance, the patients were encouraged to continuously enhance the signal intensity of action muscles, and at the same time, the intensity of antagonistic muscles was reduced, so that the normal ratio of the two was restored to 10:1, and the elbow extension function was improved. Therefore, the motion program signal derived from the digital display is an important external condition for reconstructing the motor program (see Figures 1-3). Targeted repetitive training is an important process for reconstructing the motor program.

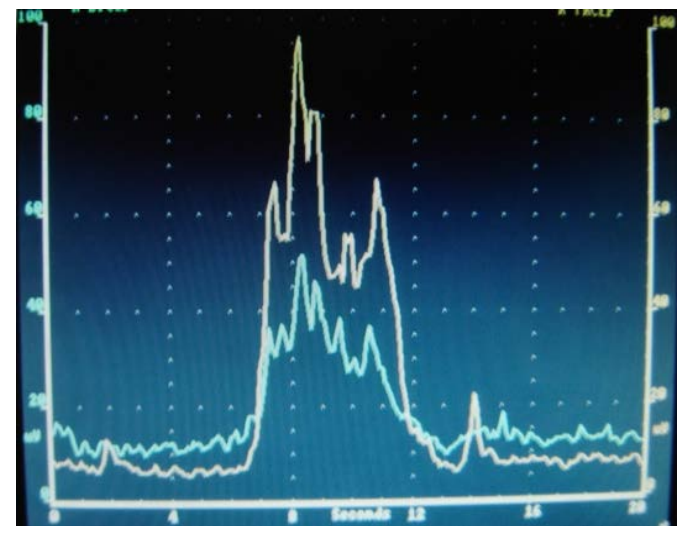

Figure 1. Right elbow extension abnormal exercise procedure: the blue signal line is the signal intensity from the exercise center to the triceps brachii, and the yellow signal line is the signal intensity to the biceps brachii. Yellow signal is too strong, elbow extension dysfunction.

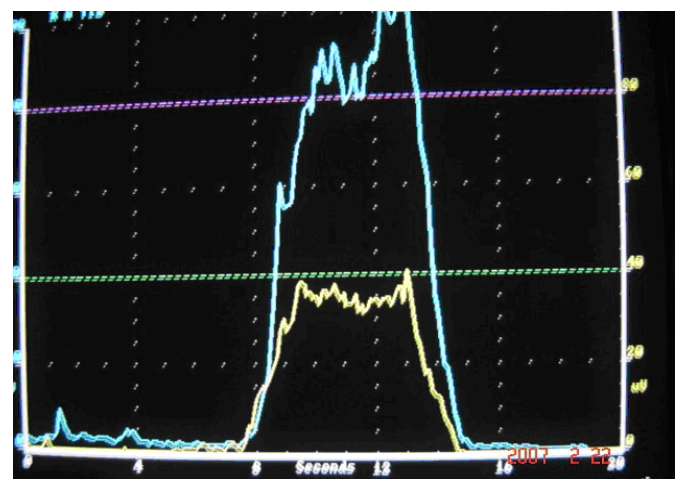

Figure 2. According to the principle that shooting at the target can improve the accuracy of archery, the physical therapist uses six step guidance to guide patients to enhance the height of blue signal line and reduce the height of yellow signal. 


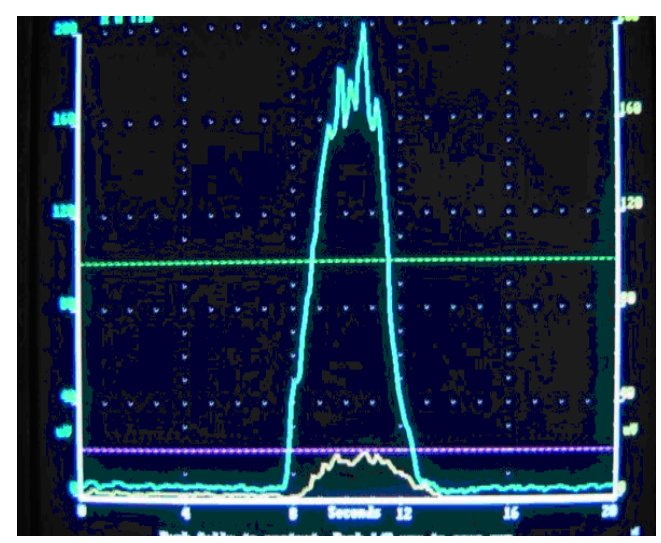

Figure 3. Through repeated training, the blue signal line is significantly improved, the yellow signal line is significantly reduced, and the abnormal exercise program is corrected. After repeated training, it is solidified, and the elbow extension function is significantly improved.

The time needed to reconstruct the motion program is related to the evolution degree of the species. The higher the evolution degree, the longer the time needed to establish the program, otherwise, the shorter. For example, sheep can walk and run within hours of birth, while it takes a year or more for a person to be able to walk and run. Therefore, it is a relatively long process to reconstruct the motor program in human brain. It needs repeated training to reconstruct and solidify the motor program. Too short a course of treatment will often make the disease repeated or even invalid. After clinical practice, we found that if the lower extremity movement dysfunction caused by CNS injury in this group generally takes 3 months of training to appear obvious functional recovery, while the upper extremity, especially the hand, takes 6 months or even longer to obtain obvious functional improvement. So that, we suggest that the course of rehabilitation training for these patients with sequelae should be $3-6$ months, which is also the basis of 90 rehabilitation training courses in 3 months in this study.

\subsection{Traditional Chinese Medicine Daoyin Feedback Technology Realizes the Symbiotic Integration of Chinese and Western Medicine Rehabilitation Technology}

In this therapy, the main therapeutic effect is to develop the potential of CNS by Daoyin operation. The technology of effect real-time display and signal feedback provides patients with the visibility target during training, so that they can accurately understand the actual situation of the motor program, and carry out accurate correction and reconstruction training, which plays an indispensable role. Therefore, the Daoyin and feedback technology of traditional Chinese medicine is the crystallization of symbiotic fusion of rehabilitation technology of traditional Chinese and Western medicine.

\section{5. "Neurological Training" [9] Is a Summary of the Daoyin Technique and Feedback Technology of Traditional Chinese Medicine}

Because of the use of traditional Chinese Medicine Daoyin technique combined with biofeedback technology to train the CNS, that is, the CNS appears plasticity changes under the guidance of feedback training, and finally achieves the process of functional reorganization and recovery of lost motor function. Therefore, we call it "neurological training" to highlight the characteristics of the integration and symbiosis of Chinese and Western rehabilitation technology.

\subsection{The Main Component of the Signal Received from the Skin Surface at the Corresponding Part of the Muscle Abdomen Is the Motor Program Signal}

Due to the complexity of neuromuscular system, many related characteristics of electromyographic 
signals such as anatomy and physiology have not been clearly understood [10] [11] [12]. Whether the signals received from the skin surface are the control or driving signals from the cortex motor center to the muscle, or the electrical activity generated by the muscle contraction itself, has not been clearly described. It is generally considered as the bioelectrical signal during the activity of neuromuscular system [13]. As the signal is received from the skin surface, it is called surface EMG signal, which is composed of action potentials of different motor units [14], and motor units are composed of a group of muscle fibers controlled by the same motor nerve.

We detected EMG signal in patients with spinal cord shock and complete peripheral nerve injury, and found that no EMG activity was detected during passive muscle movement. The irregular EMG signal measured during muscle spasm may be emitted by motoneurons in the anterior horn of the spinal cord. In the actual clinical treatment, we found that the strongest electric signals that adults and children can receive from the skin surface of the corresponding muscle parts are basically the same, no more than $640 \mathrm{uv}$. But under the same electric signal intensity, the muscle strength between them is quite different. It can be seen from one side that the electrical signals received from the skin surface mainly come from the driving signals from the central nerve to the muscles. The main function of the muscle groups involved in joint activities is the action muscle and the antagonistic muscle. The proportion of the appropriate motion program signal from the motor center to the two is the basis of joint autonomous and flexible movement. The movement of a limb needs the participation of many joints and muscles. This requires the motor center to give instructions according to the sequence of joint activities and the ratio of joint activities in the same proportion, this process is the motor program of a coordinate movement. Therefore, the signals received from the skin surface during the joint movement should be the driving electric signals from the cortex motor center to the muscle, rather than generated by the muscle contraction itself, which has been preliminarily confirmed by the animal experimental research [15].

The results of this study show that the traditional Chinese Medicine Daoyin feedback rehabilitation technology is an effective method to improve the elbow extension function of stroke patients with hemiplegia, which can better develop the potential of the central nervous system and promote the reestablishment of the motor program. Neurological training [9] is a theory based on the plasticity of the CNS and the reconstruction of the motor program. It induces the neural potential with the Daoyin technique of traditional Chinese medicine. It detects the potential signal, i.e. the motor program signal, with the bioelectrical signal receiving equipment, and displays the signal on the screen in the form of curve. Through the biofeedback mechanism, it guides the patients to control the signal intensity in a targeted way, regulate the disordered motor program and resolidify it to restore the self coordinated movement function.

\section{ACKNOWLEDGEMENTS}

During the completion of this work, support of: 1) "National Technology Support Planning Subject" is given by the ministry of science and technology of the People's Republic of China, subject number: 2013BAI10B06. 2) Project support of "China book promotion program" by the Foreign Promotion Bureu of Information Office of State Council of People's Republic of China. Number: 2016-42. 3) Science and technology project of Daxing District Integrated Hospital of traditional Chinese and Western medicine, No. kt20190230003.

\section{CONFLICTS OF INTEREST}

The authors declare no competing interests.

\section{AUTHOR'S CONTRIBUTIONS}

1) Wenru Zhao has conceived this research, by referring to file and looking up new retrieval, raises the deep mechanism of Chinese Medicine Daoyin feedback rehabilitation technique and writes this thesis. 2) Pengchuan Zhang helps to write and proofread papers. Supplementary materia. 3) Ming Qi participates 
in treatment, observe patients, collect data, and make statistical analysis; 4) Yuru Song participates in treatment and collect data.

\section{REFERENCES}

1. Wu, Z.S., Yao, C.H. and Zhao, D. (2003) Epidemiological Study on Incidence and Mortality of Stroke in Chinese Population. Chinese Journal of Epidemiology, 24, 236-239.

2. Miao, H.S. (2000) Principles and Practice of Rehabilitation Medicine. Shanghai Science and Technology Press, Shanghai, 8-13.

3. Zhao, W.-R., Zhao, H.-H., Zhang, X.-M., Cao, X. and Li, X. (2012) The Mechanism and Clinical Effectiveness of the Six-Step Chinese Daoyin Technique on Hemiplegic Patients with Drop-Foot. Journal of Biomedical Science and Engineering, 5, 652-656. https://doi.org/10.4236/jbise.2012.511081

4. Duus, P., Liu, Z.H. and Hu, W.Y. (2000) Neurolocation Diagnostics-Anatomy, Physiology, Clinical (Translated by Duan, G.S.). Naval Press, Beijing, 177.

5. Guo, B. (Translator) (2002) Adams Victor Neurology. 7th Edition, People's Health Press, Beijing, 53-55.

6. Duus, P., Liu, Z.H. and Hu, W.Y. (2000) Neurolocation Diagnostics-Anatomy, Physiology, Clinical (Translated by Duan, G.S.). Naval Press, Beijing, 287.

7. Lu, M.-K., Shih, H.-T., Huang, K.-J., et al. (2008) Movement-Related Cortical Potentials in Patients with Machado-Joseph Disease. Clinical Neurophysiology, 119, 1010-1019.

8. Dai, H. (2004) Rehabilitation Medicine. Peking University Medical Press, Beijing, 26.

9. Zhao, W.R. (2019) Rehabilitation Therapeutics of the Neurological Training: Daoyin Technique in Chinese Medicine. Springer Science + Business Media, Singapore Pte Ltd., Singapore.

10. Enoka, R.M. and Fuglevand, A.J. (2000) Motor Unit Physiology: Some Unresolved Issues. Muscle \& Nerve, 24, 4-7. https://doi.org/10.1002/1097-4598(200101)24:1<4::AID-MUS13>3.0.CO;2-F

11. McGill, K.C. (2004) Surface Electromyogram Signal Modeling. Medical and Biological Engineering and Computing, 42, 446-454. https://doi.org/10.1007/BF02350985

12. Li, Q., Yang, J.H., Chu, X.Z., Chen, X. and Zhang, X. (2007) Simulation of sEMG Signal Based on Motor Neuron Excitation. Beijing Biomedical Engineering, 26, 342-351.

13. Wang, J. and Jin, X.G. (2000) Analysis and Application of Surface Electric Signal. China Sports Science and technology, 36, 26-28.

14. Chen, X., Yang, H.J., Liang, Z., Zheng, F., Qian, X.J. and Feng, H.Q. (2003) Classification of Action Potential of NEMG Signal Motor Unit. Journal of University of Science and Technology of China, 33, 466-472.

15. Li, X., Zhao, H.H., Sun, A.P., Zhang, X.M. Cao, X. and Zhao, W.R. (2011) Sural Surface of Rabbit Experimental Study on the Source of EMG Signal. China Tissue Engineering Research and Clinical Rehabilitation, 15, 8693-8697.

\section{ABBREVIATIONS}

CNS: Central nervous system. 